\title{
Nonlinear Transient Behavior of a Piezothermoelastic Laminated Beam Subjected to Mechanical, Thermal and Electrical Load*
}

\author{
Yasuhiro WATANABE ${ }^{* *}$, Masayuki ISHIHARA ${ }^{* *}$ and Naotake NODA ${ }^{* *}$ \\ **Shizuoka University \\ 3-5-1 Johoku, Hamamatsu, Shizuoka 432-8561, Japan \\ E-mail: tmmishi@ipc.shizuoka.ac.jp
}

\begin{abstract}
Nonlinear transient behavior of a piezothermoelastic laminate including the dynamic deformation deviated arbitrarily from the equilibrium state is analyzed. As an analytical model, we consider a laminated beam composed of the elastic structural layers and the piezoelectric layers subjected to mechanical, thermal and electrical loads as disturbances or intended control procedures. The deformation of the beam is analyzed by the classical laminate theory and the von Kárman strain. Equations of motion in terms of displacements are obtained and they are analyzed through the Galerkin method. As a result, the dynamic deflection of the beam is found to be governed by the equation for a polynomial oscillator, and the following quantities are obtained: (1) the buckling temperature due to in-plane thermal and electrical loads; (2) the static large deflection due to combined in-plane and anti-plane loads; (3) the steady vibration deviated from the static equilibrium state; (4) transient large deformation with damping effect considered due to mechanical, thermal and electrical loads. Through these results, the characteristics of the transient deformation of the beam is discussed in detail.
\end{abstract}

Key words: Piezothemoelastic Laminate, Nonlinear Dynamic Behavior, Damping

\section{Introduction}

Piezoelectric materials have been extensively used as sensors and actuators to control structural configuration and to suppress undesired vibration in engineering because of their superior coupling effect between elastic and electric fields. Meanwhile, fiber reinforced plastics (FRP) such as graphite/epoxy are lighter than general metals and have the high specific strength. Moreover, lamination design of FRP is possible by compounding the various angle of fiber orientation of unidirectional reinforcement. Recently, FRP becomes promising material for light-weight structures. Sensors and actuators made of piezoelectric materials can be embedded easily in FRP structures. These structures composed of laminated FRP and piezoelectric materials are called piezothermoelastic laminates. Piezothermoelastic laminates are superior to the conventional structures composed of hydraulic actuators and acceleration sensor in that they can respond fast and stably. Therefore, piezothermoelastic laminates have attracted considerable attention in the aerospace engineering, MEMS fields, and so forth. In aerospace applications, the structures are requested to be greater and lighter. In that case, they are vulnerable to the disturbance just as environmental temperature change and the collision of space debris. 
As a result, their deformation should be relatively large. Therefore, it is important to analyze large deformation of piezothemoelastic laminates and to investigate the manner to control the undesired deformation. For example, Mukherjee and Chaudhuri ${ }^{(1)}$ analyzed static large deformation of a piezothermoelastic laminated beam and Shen ${ }^{(2)}$ analyzed postbuckling behavior of a rectangular piezothermoelastic laminate and of a piezothermoelastic cylindrical shell ${ }^{(3)}$.

Studies mentioned above ${ }^{(1-3)}$ treated static behavior of piezothermoelastic laminates. However, aerospace applications of the laminates involve dynamic deformation. Therefore, dynamic problems for large deformation of piezothermoelastic laminates were studied: Tzou and Zhou ${ }^{(4,5)}$ studied dynamic deformation of circular laminates, while Ishihara and Noda ${ }^{(6,7)}$ analyzed dynamic deformation of a rectangular laminate with geometrical nonlinearity introducing the von Kármán strains. In these studies ${ }^{(4-7)}$, the natural frequency of the oscillation in the vicinity of the static equilibrium state was obtained as the dynamic behavior.

In the analysis aforementioned ${ }^{(4-7)}$ the dynamic deformation deviating arbitrarily from the equilibrium state was not treated although it is very important from the practical viewpoint in aerospace application, for example. Therefore, Ishihara and Noda analyzed the steady vibration deviating from the equilibrium state ${ }^{(8)}$ and obtained the relation between the deflection of the laminate and its velocity under various loading conditions excluding the effect of damping.

From more practical viewpoints, it is important to consider the effect of damping on analyzing the dynamic behavior because damping changes such dynamic characters as the transient behavior, natural frequencies and so forth. Therefore, the analysis of transient dynamic behavior taking damping into account is important to estimate dynamic characteristics properly. In this paper, therefore, we analyze the nonlinear transient dynamic behavior of a piezothermoelastic laminated beam with damping effect considered, including the dynamic deflection deviating arbitrarily from the equilibrium state. As an analytical model, we consider a laminated beam composed of fiber-reinforced laminate and piezoelectric layers subjected to mechanical, thermal and electrical loads as disturbances or as intended control procedures with both ends simply-supported. Nonlinear large deformation of the laminate is analyzed based on the von Kármán strain ${ }^{(9)}$ and the classical laminate theory. Equations of motion of the beam are analyzed using the Galerkin $\operatorname{method}^{(10)}$. As a result, it is found that the dynamic deflection of the beam is governed by the equation for a polynomial oscillator ${ }^{(11)}$. According to the equation, the following quantities are obtained: (1) the buckling temperature due to in-plane thermal and electrical load; (2) the static large deflection due to combined in-plane and anti-plane loads; (3) the steady vibration deviated arbitrarily from the static equilibrium state; (4) transient large deformation with damping effect considered due to mechanical, thermal and electrical loads. Moreover, numerical calculation is performed to investigate the nonlinear transient deformation and obtain the manner to stabilize it.

\section{Theoretical Analysis}

\subsection{Problem}

Consider a simply-supported beam with dimension $a \times b \times h$ composed of $N$ layers as shown in Fig.1. Two of $N$ layers $\left(z_{k-1} \leq z \leq z_{k}, z_{k^{\prime}-1} \leq z \leq z_{k^{\prime}}\right)$ exhibit piezoelectricity while other layers do not. The beam is a cross-ply laminate: all the layers are orthotropic and the principal axes of orthotropy coincide with the axes of the Cartesian coordinate system $(x, y, z)$. The layers are laminated symmetrically with respect to the central plane $z=0$, that is, the $i$-th and $(N-i+1)$-th layers are composed of the same 
material and have same orthotropy with respect to the Cartesian coordinate system $(x, y, z)$.

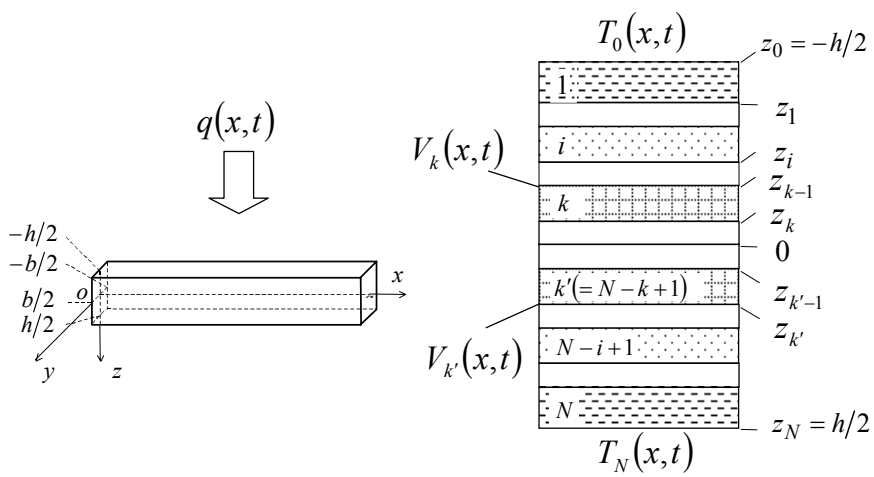

Fig.1 Analytical model.

The laminate is subjected to transverse load $q$ in $z$-direction and temperature $T_{0}$ and $T_{N}$ on the upper $(z=-h / 2)$ and the lower $(z=h / 2)$ surfaces of the laminate, respectively, as mechanical and thermal disturbances. To control the effects of the transverse load and the temperature, it is also subjected to electric potential $V_{k}$ and $V_{k^{\prime}}$ on the upper surface $\left(z=z_{k-1}\right)$ of the $k$-th layer and the lower surface $\left(z=z_{k^{\prime}}\right)$ of the $k^{\prime}$-th layer, respectively. The lower surface $\left(z=z_{k}\right)$ of the $k$-th layer and the upper surface $\left(z=z_{k^{\prime}-1}\right)$ of the $k^{\prime}$-th layer are both the level surfaces of electric potential.

\subsection{Governing Equations}

Since the thickness of the beam are much smaller than the length of the beam, the Kirchhoff's hypothesis is assumed to be valid, that is, the displacement components in the $x$ - and $z$-directions are respectively taken as:

$$
u=u^{0}(x, t)-z \frac{\partial w(x, t)}{\partial x}, w=w(x, t),
$$

where the superscript 0 denotes the quantity at the central plane $(z=0)$. The assumption to treat nonlinear deformation is introduced. Since the inplane dimension are much larger than the antiplane dimension, the inplane strain and the square of the rotation about $y$ axis are assumed to be small in the same order compared to unity, that is, the von Kármán strain is introduced for the normal strain in the $x$-direction as:

$$
\varepsilon_{x x}=\frac{\partial u}{\partial x}+\frac{1}{2}\left(\frac{\partial w}{\partial x}\right)^{2}
$$

Quasi-static electric field in $z$-direction $E_{z}$ is expressed by electric potential $\phi$ as

$$
E_{z}=-\frac{\partial \phi}{\partial z}
$$

Assuming the normal stresses in $y$ - and $z$-directions are negligible, the constitutive equations of piezothermoelasticity ${ }^{(8)}$ are reduced to one-dimensional form as:

$$
\sigma_{x x}=c_{i} \varepsilon_{x x}-e_{i} E_{z}-\lambda_{i} T
$$

where $\sigma_{x x}$ denotes the normal stress in $x$-direction; $T$ denotes temperature; $c_{i}, e_{i}$ and $\lambda_{i}$ denote elastic stiffness coefficient, piezoelectric coefficient and stress-temperature coefficient for $i$-th layer. The resultant force $N_{x}$ and the resultant moment $M_{x}$ are defined as

$$
N_{x} \equiv \int_{-h / 2}^{h / 2} \sigma_{x x} \mathrm{~d} z, \quad M_{x} \equiv \int_{-h / 2}^{h / 2} \sigma_{x x} z \mathrm{~d} z
$$


Substitution of Eqs.(1),(2)and(4) into Eq.(5) gives

$$
N_{x}=A\left[\frac{\partial u^{0}}{\partial x}+\frac{1}{2}\left(\frac{\partial w}{\partial x}\right)^{2}\right]-N_{x}^{T}-N_{x}^{E}, M_{x}=-D \frac{\partial^{2} w}{\partial x^{2}}-M_{x}^{T}-M_{x}^{E}
$$

where the definitions of $A, D, N_{x}^{T}, M_{x}^{T}, N_{x}^{E}$ and $M_{x}^{E}$ are given as

$$
\begin{aligned}
& \{A, D\}=\sum_{i=1}^{N} \int_{z_{i-1}}^{z_{i}} c_{i}\left\{1, z^{2}\right\} \mathrm{d} z \\
& \left\{N_{x}^{T}, M_{x}^{T}\right\}=\sum_{i=1}^{N} \int_{z_{i-1}}^{z_{i}} \lambda_{i} T\{1, z\} \mathrm{d} z,\left\{N_{x}^{E}, M_{x}^{E}\right\}=\sum_{i=1}^{N} \int_{z_{i-1}}^{z_{i}} e_{i} E_{z}\{1, z\} \mathrm{d} z .
\end{aligned}
$$

Equations of motion which integrate the effect of in-plane resultant forces into anti-plane motion are given as follows:

$$
\frac{\partial N_{x}}{\partial x}=0, \quad P \frac{\partial^{2} w}{\partial t^{2}}+c_{d} \frac{\partial w}{\partial t}=N_{x} \frac{\partial^{2} w}{\partial x^{2}}+\frac{\partial^{2} M_{x}}{\partial x^{2}}+q
$$

where $c_{d}$ denotes the damping coefficient and $P$ is defined by mass density of $i$-th layer $\rho_{i}$ as

$$
P=\sum_{i=1}^{N} \int_{z_{i-1}}^{z_{i}} \rho_{i} \mathrm{~d} z
$$

By substituting Eq.(6) into Eq.(9), the equations of motion expressed by the displacements are given as follows:

$$
\left.\begin{array}{l}
L_{1}\left(u^{0}, w\right)=\frac{\partial}{\partial x}\left(N_{x}^{T}+N_{x}^{E}\right), \\
P \frac{\partial^{2} w}{\partial t^{2}}+c_{d} \frac{\partial w}{\partial t}+L_{2}\left(u^{0}, w\right)=q-\frac{\partial^{2}}{\partial x^{2}}\left(M_{x}^{T}+M_{x}^{E}\right)-\left(N_{x}^{T}+N_{x}^{E}\right) \frac{\partial^{2} w}{\partial x^{2}}
\end{array}\right\}
$$

where the differentiation operators $L_{1}$ and $L_{2}$ are defined as

$$
L_{1}\left(u^{0}, w\right)=A\left[\frac{\partial^{2} u^{0}}{\partial x^{2}}+\frac{\partial w}{\partial x} \frac{\partial^{2} w}{\partial x^{2}}\right], L_{2}\left(u^{0}, w\right)=-A\left[\frac{\partial u^{0}}{\partial x}+\frac{1}{2}\left(\frac{\partial w}{\partial x}\right)^{2}\right] \frac{\partial^{2} w}{\partial x^{2}}+D \frac{\partial^{4} w}{\partial x^{4}} .
$$

As the beam is simply-supported, we have

$$
x=0, a ; \quad u^{0}=0, w=0, M_{x}=-D \frac{\partial^{2} w}{\partial x^{2}}-\left(M_{x}^{T}+M_{x}^{E}\right)=0 .
$$

\subsection{Galerkin Method}

We use the Galerkin method ${ }^{(10)}$ to solve the governing equations(11) under the boundary conditions (13). We choose trigonometric functions as the trial functions and consider displacements are expressed by series as

$$
\left\{u^{0}, w\right\}=\sum_{m=1}^{\infty}\left\{u_{m}(t), w_{m}(t)\right\}_{\sin } \alpha_{m} x: \alpha_{m}=\frac{m \pi}{a}
$$

so that the boundary conditions (13) may be satisfied.

First, we decide the thermally-induced resultant force $N_{x}^{T}$ and moment $M_{x}^{T}$. Hereafter, we consider that $q, T_{0}, T_{N}, V_{k}$ and $V_{k^{\prime}}$ are uniform with respect to variable $x$ as follows:

$$
q(x, t) \equiv Q(t), T_{0}(x, t) \equiv \Theta_{0}(t), T_{N}(x, t) \equiv \Theta_{N}(t), V_{k}(x, t) \equiv V_{k}(t), V_{k^{\prime}}(x, t) \equiv V_{k^{\prime}}(t) .
$$

Considering the length of the beam is sufficiently larger than the thickness of the 
beam $(a>>h)$, the distribution of the temperature can be regarded to be linear with respect to $z$. Therefore, $N_{x}^{T}$ and $M_{x}^{T}$ are obtained from Eq.(8) expressed as follows:

$$
\left.\begin{array}{l}
N_{x}^{T}=h \cdot \frac{1}{2}\left[\Theta_{N}(t)+\Theta_{0}(t)\right] \sum_{i=1}^{N} \lambda_{i}\left(\frac{z_{i}}{h}-\frac{z_{i-1}}{h}\right), \\
M_{x}^{T}=h^{2} \cdot\left[\Theta_{N}(t)-\Theta_{0}(t)\right] \sum_{i=1}^{N} \lambda_{i} \frac{1}{3}\left[\left(\frac{z_{i}}{h}\right)^{3}-\left(\frac{z_{i-1}}{h}\right)^{3}\right]
\end{array}\right\} .
$$

Second, we decide the electrically-induced resultant force $N_{x}^{E}$ and moment $M_{x}^{E}$. Considering that the length of the beam is sufficiently larger than the thickness of the each piezoelectric layer and that the distribution of electric potential can be regarded to be linear with respect to $z$ in each piezoelectric layer, therefore, $N_{x}^{E}$ and $M_{x}^{E}$ are obtained from Eq.(8) as follows:

$$
N_{x}^{E}=e_{k}\left[V_{k}(t)-V_{k^{\prime}}(t)\right], \quad M_{x}^{E}=-e_{k}\left[V_{k}(t)+V_{k^{\prime}}(t)\right] \frac{z_{k}+z_{k-1}}{2} .
$$

Then, from Eqs.(3)and(8), it is found that the thermally-induced resultant force $N_{x}^{T}$ and the electrically-induced resultant force $N_{x}^{E}$ become uniform with respect to variable $x$. On the other hand, in order to satisfy the boundary conditions (13), the transverse load $q$, the thermally-induced resultant moment $M_{x}^{T}$ and the electrically-induced resultant moment $M_{x}^{E}$ are evaluated as

$$
\begin{aligned}
& \left\{Q, M_{x}^{T}, M_{x}^{E}\right\}=\sum_{m=1}^{\infty}\left\{Q_{m}(t), M_{x, m}^{T}(t), M_{x, m}^{E}(t)\right\} \sin \alpha_{m} x, \\
& \left.\left\{Q_{m}(t), M_{x, m}^{T}(t), M_{x, m}^{E}(t)\right\}=\frac{2}{a} \int_{0}^{a}\left\{Q(t), M_{x}^{T}, M_{x}^{E}\right\} \sin \alpha_{m} x \mathrm{~d} x\right\} .
\end{aligned}
$$

Substitution of Eqs.(16)and(17) into Eq.(18) gives

$$
\left.\begin{array}{l}
Q_{m}(t)=Q(t) \cdot \frac{4}{m \pi} \cdot \bmod (m, 2), \\
M_{x, m}^{T}(t)=h^{2} \cdot\left[\Theta_{N}(t)-\Theta_{0}(t)\right] \sum_{i=1}^{N} \lambda_{i} \frac{1}{3}\left[\left(\frac{z_{i}}{h}\right)^{3}-\left(\frac{z_{i-1}}{h}\right)^{3}\right] \cdot \frac{4}{m \pi} \cdot \bmod (m, 2), \\
M_{x, m}^{E}(t)=e_{k}\left[V_{k}(t)+V_{k^{\prime}}(t)\right] \cdot \frac{z_{k}+z_{k-1}}{2} \cdot \frac{4}{m \pi} \cdot \bmod (m, 2)
\end{array}\right\} .
$$

Then, applying the Galerkin method to Eq.(11), we have

$$
\left.\begin{array}{l}
\int_{0}^{a}\left[L_{1}\left(u^{0}, w\right)-\frac{\partial}{\partial x}\left(N_{x}^{T}+N_{x}^{E}\right)\right] \sin \alpha_{m^{\prime}} x \mathrm{~d} x=0 \\
\int_{0}^{a}\left[P \frac{\partial^{2} w}{\partial t^{2}}+c_{d} \frac{\partial w}{\partial t}+L_{2}\left(u^{0}, w\right)-Q+\frac{\partial^{2}\left(M_{x}^{T}+M_{x}^{E}\right)}{\partial x^{2}}+\left(N_{x}^{T}+N_{x}^{E}\right) \frac{\partial^{2} w}{\partial x^{2}}\right] \sin \alpha_{m^{\prime}} x \mathrm{~d} x=0 \\
: m^{\prime}=1,2,3, \ldots, \infty .
\end{array}\right\}
$$

By substituting Eqs.(12),(13)and(18) into Eq.(20) and performing integrations in Eq.(20), we have the simultaneous nonlinear equations with respect to $u_{m}$ and $w_{m}$ as follows: 


$$
\begin{aligned}
& \alpha_{m}^{2} u_{m}+\sum_{m^{\prime}=1}^{\infty} \sum_{i=1}^{\infty} \frac{1}{2} \alpha_{m^{\prime}}^{2} \alpha_{i} w_{m^{\prime}} w_{i} \delta_{m^{\prime} i m}=0 \\
& P \frac{d^{2} w_{m}}{d t^{2}}+c_{d} \frac{d w_{m}}{d t}+\left[D \alpha_{m}^{4}-\alpha_{m}^{2}\left(N_{x}^{T}+N_{x}^{E}\right)\right] w_{m}+\sum_{m^{\prime}=1}^{\infty} \sum_{i=1}^{\infty} \frac{1}{2} A \alpha_{m^{\prime}}^{2} \alpha_{i} \delta_{m^{\prime} i m} w_{m^{\prime}} u_{i} \\
& \quad+\sum_{m^{\prime}=1}^{\infty} \sum_{i=1}^{\infty} \sum_{k=1}^{\infty} \frac{1}{8} \alpha_{m^{\prime}}^{2} \alpha_{i} \alpha_{k} \Delta_{c, i k m^{\prime} m} w_{m^{\prime}} w_{i} w_{k}=Q_{m}+\alpha_{m}^{2}\left(M_{x, m}^{T}+M_{x, m}^{E}\right) \\
& : \quad m^{\prime}=1,2,3, \ldots, \infty .
\end{aligned}
$$

where the definitions of $\delta_{i j k}$ and $\Delta_{c, i j k l}$ are given in the previous paper ${ }^{(8)}$. Then, by eliminating $u_{m}$ from Eq.(21), we have the simultaneous nonlinear ordinary differential equations with respect to $w_{m}(m=1,2,3, \ldots, \infty)$ as follow:

$$
\begin{aligned}
& (P h) \frac{\mathrm{d}^{2}}{\mathrm{~d} t^{2}}\left(\frac{w_{m}}{h}\right)+\left(c_{d} h\right) \frac{\mathrm{d}}{\mathrm{d} t}\left(\frac{w_{m}}{h}\right)+k_{m}^{L}\left(\frac{w_{m}}{h}\right)+\sum_{m^{\prime}=1}^{\infty} \sum_{i=1}^{\infty} \sum_{k=1}^{\infty} k_{m, m^{\prime} i k}^{N}\left(\frac{w_{m^{\prime}}}{h}\right)\left(\frac{w_{i}}{h}\right)\left(\frac{w_{k}}{h}\right)=p_{m} \\
& : m=1,2,3, \ldots, \infty
\end{aligned}
$$

where the definitions of $k_{m}^{L}, k_{m, m^{\prime} i k}^{N}$ and $p_{m}$ are given as

$$
\left.\begin{array}{l}
k_{m}^{L}\left(=k_{m}^{L}(t)\right)=h \alpha_{m}^{2}\left[D \alpha_{m}^{2}-\left(N_{x}^{T}+N_{x}^{E}\right)\right] \\
k_{m, m^{\prime} i k}^{N}=\frac{1}{8} h^{3} A \alpha_{m^{\prime}}^{2} \alpha_{i} \alpha_{k}\left(\Delta_{c, i k m^{\prime} m}-\sum_{l=1}^{\infty} \frac{2 \alpha_{i}}{\alpha_{l}} \delta_{i k l} \delta_{m^{\prime} l m}\right) \\
p_{m}\left(=p_{m}(t)\right)=Q_{m}+\alpha_{m}^{2}\left(M_{x, m}^{T}+M_{x, m}^{E}\right)
\end{array}\right\} .
$$

\subsection{Polynomial Oscillator}

To develop the physical characteristics of the dynamic behavior of the beam, we simplify Eq.(22). By truncating infinite series in Eq.(14), therefore, in Eq.(22), up to one term, equation(22) for $m=1$, we have the nonlinear equation with respect to $w_{1}$ as follows:

$$
(P h) \frac{\mathrm{d}^{2}}{\mathrm{~d} t^{2}}\left(\frac{w_{1}}{h}\right)+\left(c_{d} h\right) \frac{\mathrm{d}}{\mathrm{d} t}\left(\frac{w_{1}}{h}\right)+k_{1}^{L}\left(\frac{w_{1}}{h}\right)+k_{1,111}^{N}\left(\frac{w_{1}}{h}\right)^{3}=p_{1} .
$$

Note that, from Eq.(14), $w_{1}$ denotes the deflection at the center of the laminate $(x=a / 2)$. By introducing non-dimensional variables as

$$
U(=U(\tau)) \equiv \frac{w_{11}}{h}, \tau \equiv \sqrt{\frac{k_{1,111}^{N}}{P h}} t, \lambda(=\lambda(\tau)) \equiv-\frac{k_{1}^{L}}{k_{1,111}^{N}}, \delta \equiv \frac{\left(c_{d} h\right)}{\sqrt{k_{1,111}^{N}(P h)}}, \alpha(=\alpha(\tau)) \equiv \frac{p_{1}}{k_{1,111}^{N}} .
$$

Equation(24) is rewritten in non-dimensional form as

$$
\frac{\mathrm{d}^{2} U}{\mathrm{~d} \tau^{2}}+\delta \frac{\mathrm{d} U}{\mathrm{~d} \tau}-\lambda U+U^{3}=\alpha \quad: \quad \delta \geq 0
$$

or in dynamical system as

$$
\frac{\mathrm{d} U}{\mathrm{~d} \tau}=V, \frac{\mathrm{d} V}{\mathrm{~d} \tau}=\lambda U-U^{3}-\delta V+\alpha: \delta \geq 0 .
$$

Note that $\alpha \geq 0$ in Eqs.(26)and(27) does not lose generality because $(U, V)$ for $\alpha=\alpha_{0}(<0)$ is obtained as $(-U,-V)$ for $\alpha=-\alpha_{0}(>0)$. Thus, from Eqs.(26)and(27), it is found that the dynamic deflection of the laminate $(U)$ is governed by the equation for the polynomial oscillator ${ }^{(11)}$ with three parameters $\lambda, \delta$ and $\alpha$. 


\subsection{Physical Characteristics of Dynamic Behavior}

\subsubsection{Static large deflection}

At first, let us examine the static large deflection of the laminate and its stability. The static large deflection is obtained as the equilibrium point of the dynamical system described by Eq.(27). Letting $\left(U_{e}, V_{e}\right)$ be the equilibrium point of $(U, V),\left(U_{e}, V_{e}\right)$ is obtained as the solution of the following equations:

$$
0=V_{e}, \quad 0=\lambda U_{e}-U_{e}^{3}-\delta V_{e}+\alpha .
$$

Equation(28) is an algebraic cubic equation and the solution is obtained for $\alpha=0$ as

$$
\left.\begin{array}{l}
U_{e}=0\left(\equiv U_{1}\right) \\
U_{e}= \pm \sqrt{\lambda}\left(\equiv U_{2}\right): \lambda>0
\end{array}\right\}
$$

and for $\alpha>0$ as

$$
\begin{aligned}
& U_{e}=\sqrt[3]{\frac{\alpha}{2}+\sqrt{\left(\frac{\alpha}{2}\right)^{2}-\left(\frac{\lambda}{3}\right)^{3}}}-\sqrt[3]{-\frac{\alpha}{2}+\sqrt{\left(\frac{\alpha}{2}\right)^{2}-\left(\frac{\lambda}{3}\right)^{3}}}\left(\equiv U_{3}\right): \lambda<0 \\
& \left.U_{e}=\sqrt[3]{\frac{\alpha}{2}+\sqrt{\left(\frac{\alpha}{2}\right)^{2}-\left(\frac{\lambda}{3}\right)^{3}}}+\sqrt[3]{\frac{\alpha}{2}-\sqrt{\left(\frac{\alpha}{2}\right)^{2}-\left(\frac{\lambda}{3}\right)^{3}}}\left(\equiv U_{4}\right) \quad: 0<\lambda<3 \cdot \sqrt[3]{\left(\frac{\alpha}{2}\right)^{2}}\right\} \\
& U_{e}=2 \sqrt{\frac{\lambda}{3}} \cdot\left\{\cos \frac{\theta}{3}, \cos \frac{\theta+2 \pi}{3}, \cos \frac{\theta-2 \pi}{3}\right\}\left(\equiv\left\{U_{5}, U_{6}, U_{7}\right\}\right): \lambda>3 \cdot \sqrt[3]{\left(\frac{\alpha}{2}\right)^{2}}
\end{aligned}
$$

where

$$
\theta=\cos ^{-1}\left[\left(\frac{\alpha}{2}\right) / \sqrt{\left(\frac{\lambda}{3}\right)^{3}}\right](0 \leq \theta \leq \pi) .
$$

The stability of the deflection described by Eqs.(29)and(30) is examined by considering the deviation of $(U, V)$ from the equilibrium point $\left(U_{e}, V_{e}\right)$ as

$$
\bar{U}=U-U_{e}, \bar{V}=V-V_{e} .
$$

By substituting Eq.(32) into Eq.(27) combined with Eq.(28), the dynamical system for the deviation $(\bar{U}, \bar{V})$ is expressed by

$$
\frac{\mathrm{d} \mathbf{X}}{\mathrm{d} \tau}=\mathbf{J X}+\mathbf{N}(\mathbf{X})
$$

where

$$
\mathbf{X}=\left\{\begin{array}{l}
\bar{U} \\
\bar{V}
\end{array}\right\}, \mathbf{J}=\left[\begin{array}{cc}
0 & 1 \\
\lambda-3 U_{e}^{2} & -\delta
\end{array}\right], \mathbf{N}(\mathbf{X})=\left\{\begin{array}{c}
0 \\
-\left(3 U_{e} \bar{U}^{2}+\bar{U}^{3}\right)
\end{array}\right\} .
$$

For small deviation $(|\bar{U}|<<1,|\bar{V}|<<1)$, the system described by Eq.(33) is linearized as

$$
\frac{\mathrm{d} \mathbf{X}}{\mathrm{d} \tau}=\mathbf{J X}
$$

and the eigenvalue of Eq.(35), $s$, is obtained as

$$
s=-\frac{\delta}{2} \pm \sqrt{\left(\frac{\delta}{2}\right)^{2}-\left(-\lambda+3 U_{e}^{2}\right)} .
$$

The deflection is stable when $\operatorname{Re}(s)$ could not be positive and it is unstable when $\operatorname{Re}(s)$ could be positive ${ }^{(11)}$. By substituting Eqs.(29)and (30) into Eq.(36), it is found that $U_{1}$ is stable when $\lambda<0$ and it is unstable when $\lambda>0$, that $U_{2}, U_{3}, U_{4}, U_{5}$ and $U_{6}$ are 
stable, and that $U_{7}$ is unstable.

The variations of the static large deflection of the beam with parameter $\lambda$ for $\alpha=0$ and $\alpha>0$ are shown graphically in Fig.2, where solid lines denote stable deflection, broken lines denote unstable one. From Fig.2, first, let us examine the case of $\alpha=0$. When $\lambda$ is negative, the deflection is zero and stable. As $\lambda$ increases, deflection- $\lambda$ curve bifurcates into three curves at $\lambda=0$ : one is a zero and unstable deflection and the others are two non-zero and stable deflections. Second, let us examine the case of $\alpha>0$. A stable deflection increases with parameter $\lambda$, and its variation is smoother than the case of $\alpha=0$. Moreover, another stable deflection and an unstable one appear when $\lambda$ is greater than a positive critical value.

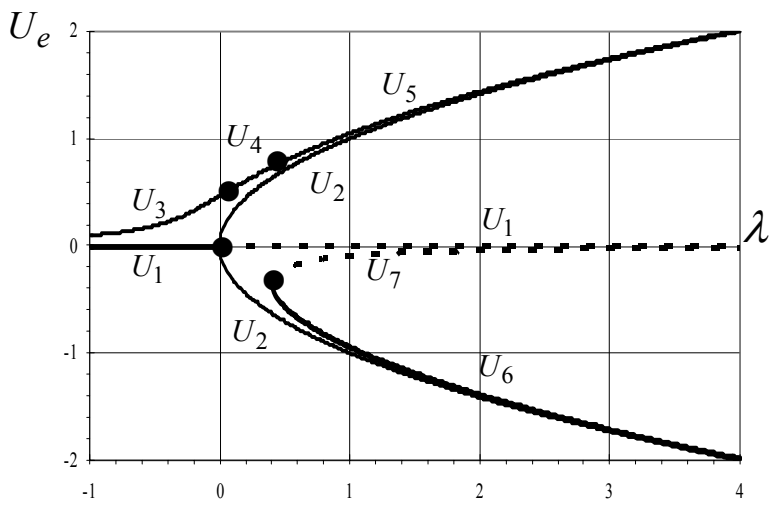

Fig.2 Variation of static large deflection with parameter $\lambda(\alpha=0.1)$

In order to connect the behavior of the dynamical system with engineering sense, let us consider the situation:

$$
Q=0, \quad \Theta_{N}=\Theta_{0}\left(\equiv \Theta_{0}\right), \quad V_{k}=-V_{k^{\prime}}(\equiv V)
$$

where $\Theta_{0}$ and $V$ are assumed as thermal disturbance and control procedure, respectively. Then, from Eqs.(16),(17),(19),(23),(25)and(37), we have

$$
\alpha=0, \quad \lambda=-\frac{h \alpha_{1}^{2}}{k_{1,111}^{N}}\left\{D \alpha_{1}^{2}-\left[\Theta_{0} \sum_{i=1}^{N} \lambda_{i}\left(z_{i}-z_{i-1}\right)+2 e_{k} V\right]\right\} .
$$

As shown in Eq.(29) and Fig.2, the beam starts to deflect at $\lambda=0$ with the increase of $\lambda$. From Eq.(38), $\lambda=0$ gives the critical temperature

$$
\Theta_{0}=\frac{D \alpha_{1}^{2}-2 e_{k} V}{\sum_{i=1}^{N} \lambda_{i}\left(z_{i}-z_{i-1}\right)}
$$

which is referred to as the buckling temperature. From Eq.(39), it is found that the buckling temperature changes linearly with respect to the voltage and that appropriate voltage to the actuators can increase the buckling temperature. From Eqs.(38)and(39), it should be noted that $\lambda>0$ means that temperature is greater than the buckling temperature.

To examine the engineering aspects of the results mentioned above, numerical examples are shown. We assume that the piezoelectric layers are of $\mathrm{BaTiO}_{3}$ and other layers are of graphite/epoxy (GE). Materials constants are given as, for GE layers ${ }^{(8)}$,

$$
\left.\begin{array}{l}
c_{i}=181[\mathrm{GPa}], \lambda_{i}=3.62 \times 10^{3}\left[\mathrm{~Pa} \cdot \mathrm{K}^{-1}\right](\text { for } x \text {-direction reinforcement }), \\
c_{i}=10.3[\mathrm{GPa}], \lambda_{i}=231.8 \times 10^{3}\left[\mathrm{~Pa} \cdot \mathrm{K}^{-1}\right](\text { for } y \text {-direction reinforcement }), \\
\rho_{i}=1.58 \times 10^{3}\left[\mathrm{~kg} \cdot \mathrm{m}^{-3}\right]
\end{array}\right\}
$$


and for $\mathrm{BaTiO}_{3}$ layer ${ }^{(8)}$,

$$
c_{i}=109[\mathrm{GPa}], \lambda_{i}=0.932 \times 10^{6}\left[\mathrm{~Pa} \cdot \mathrm{K}^{-1}\right\rfloor, \rho_{i}=5.7 \times 10^{3}\left\lfloor\mathrm{~kg} \cdot \mathrm{m}^{-3}\right\rfloor, e_{i}=-8.57\left\lfloor\mathrm{C} \cdot \mathrm{m}^{-2}\right\rfloor
$$

We assume that the dimensions of the beam are given as $a=1[\mathrm{~m}], h=0.01[\mathrm{~m}]$ and that the laminae with the same thickness are piled as $\left.\left\{\mathrm{BaTiO}_{3}: 0^{\circ}\right] /\left[\mathrm{GE}:\left(0^{\circ} / 90^{\circ}\right)_{2}\right]\right\}_{\text {sym }}$ $\left(N=10, k=1, k^{\prime}=10\right)$, where $0^{\circ}$ and $90^{\circ}$ denote $x$ - and $y$-direction reinforcement respectively. In this case, the buckling temperature for $V=0[\mathrm{~V}]$ in Eq.(37) is calculated as $\Theta_{0}\left(=\Theta_{c r}\right)=34.75[\mathrm{~K}]$.

\subsubsection{Transient large deflection}

Then, let us examine the transient large deflection deviated arbitrarily from the equilibrium state. By introducing new non-dimensional variables as

$$
U^{\prime} \equiv \frac{U}{\sqrt{|\lambda|}}, \quad \tau^{\prime} \equiv \tau \sqrt{|\lambda|}, \quad V^{\prime} \equiv \frac{d U^{\prime}}{d \tau^{\prime}}, \quad \alpha^{\prime} \equiv \frac{\alpha}{|\lambda| \sqrt{|\lambda|}}, \quad \delta^{\prime} \equiv \frac{\delta}{\sqrt{|\lambda|}},
$$

Eq.(27) is rewritten as

$$
\frac{\mathrm{d} U^{\prime}}{\mathrm{d} \tau^{\prime}}=V^{\prime}, \frac{\mathrm{d} V^{\prime}}{\mathrm{d} \tau^{\prime}}=-f\left(U^{\prime}: \alpha^{\prime}, \delta^{\prime}\right)
$$

where

$$
f\left(U^{\prime}: \alpha^{\prime}, \delta^{\prime}\right)=U^{\prime}+U^{\prime 3}-\alpha^{\prime}+\delta^{\prime} V^{\prime}
$$

for $\lambda<0$ and

$$
f\left(U^{\prime}: \alpha^{\prime}, \delta^{\prime}\right)=-U^{\prime}+U^{\prime 3}-\alpha^{\prime}+\delta^{\prime} V^{\prime}
$$

for $\lambda>0$. By solving Eq.(43) for $\left(U^{\prime}, V^{\prime}\right)$ through the Galerkin method, we analyze the transient behavior of a piezothermoelastic laminated beam and we present the results by orbits, that is, the relation between the nonlinear deflection $U^{\prime}$ and the nonlinear velocity $V^{\prime}$.

From Eqs.(29),(30)and(42), the transient behavior is classified into four cases depending on parameters $\lambda$ and $\alpha^{\prime}$ as

$$
\left.\begin{array}{lll}
\lambda<0 & & \cdots \text { case(i) } \\
\lambda>0 \text { and } \alpha^{\prime}>2 \sqrt{3} / 9 & \cdots \text { case(ii) } \\
\lambda>0 \text { and } \alpha^{\prime}=2 \sqrt{3} / 9 & \cdots \text { case(iii) } \\
\lambda>0 \text { and } 0 \leq \alpha^{\prime}<2 \sqrt{3} / 9 & \cdots \text { case(iv) }
\end{array}\right\} .
$$

In order to connect the behavior with engineering sense, let us consider the situation:

$$
Q=0, \Theta_{N}=\Theta_{0}\left(\equiv \Theta_{0}\right), V_{k}=V_{k^{\prime}}(\equiv V)
$$

where $\Theta_{0}$ and $V$ are assumed as thermal disturbance and control procedure, respectively.

Then, from Eqs.(16),(17),(19),(23),(25),(42)and(47),

$$
\alpha^{\prime}=\frac{4 \alpha_{1}^{2}\left(z_{k}+z_{k-1}\right) e_{k} V}{\pi|\lambda| \sqrt{|\lambda|} k_{1,111}^{N}}, \lambda=\frac{h \alpha_{1}^{2}}{k_{1,111}^{N}} \sum_{i=1}^{N} \lambda_{i}\left(z_{i}-z_{i-1}\right)\left(\Theta_{0}-\Theta_{c r}\right), \Theta_{c r}=\frac{D \alpha_{1}^{2}}{\sum_{i=1}^{N} \lambda_{i}\left(z_{i}-z_{i-1}\right)} .
$$

Thus, $\lambda<0$ and $\lambda>0$ means that the beam is subjected to the temperature that is lower and higher than the buckling temperature, respectively, and $\alpha^{\prime}$ corresponds to the electric voltage applied to the piezoelectric layers. For the beam considered in Subsection 2.5.1, $\Theta_{0}=10[\mathrm{~K}]$ and $V=380[\mathrm{~V}]$ in Eq.(47) gives $\alpha^{\prime}=0.1$, and for this case $U^{\prime}=1$ 
corresponds to $w_{1}=5.35[\mathrm{~mm}]$.

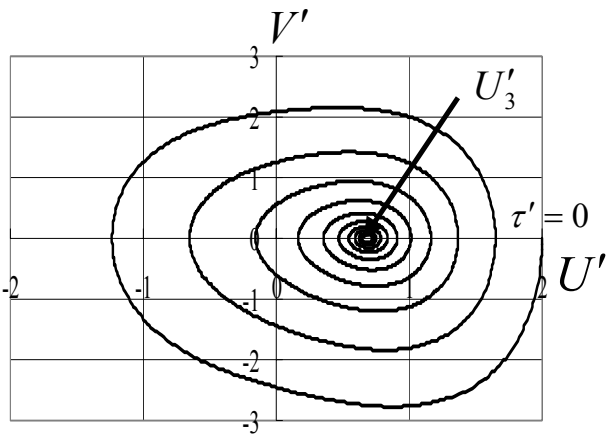

Fig.3 Transient deformation for case (i) $\left(\lambda<0, \quad \alpha^{\prime}=1, \quad \delta^{\prime}=0.2\right)$

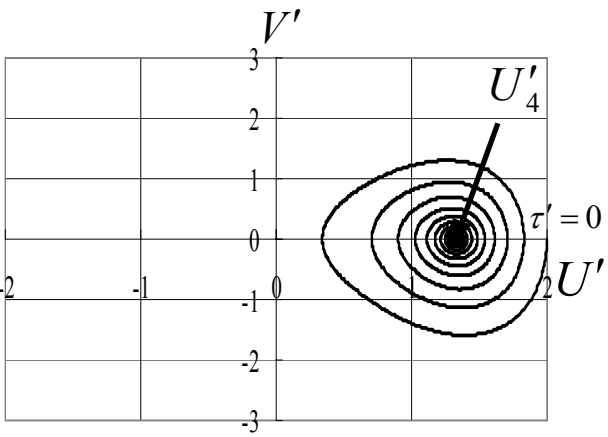

Fig.4 Transient deformation for case (ii) $\left(\lambda>0, \alpha^{\prime}=1, \delta^{\prime}=0.2\right)$

Figure 3 shows the transient vibration of the beam for the case (i), where the temperature is lower than the buckling temperature. Figure 4 shows the result for the case (ii), where the temperature is higher than the buckling temperature and the electric voltage is higher than a critical value. From Figs.3and4, it is found that the beam vibrates, decays and tends to the final deflection $U_{3}^{\prime}$ (corresponding to $U_{3}$ ) and $U_{4}^{\prime}$ (corresponding to $U_{4}$ ), respectively.

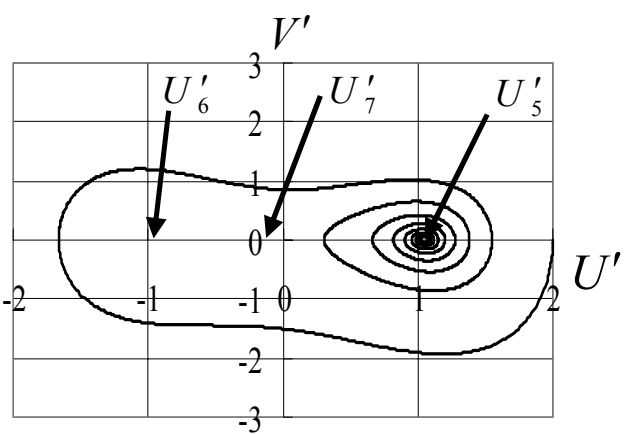

(a) $\left.\left(U^{\prime}, V^{\prime}\right)\right|_{\tau^{\prime}=0}=(2,0)$

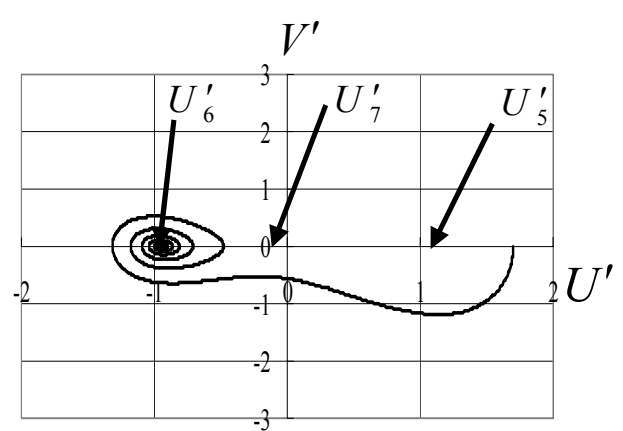

(b) $\left.\left(U^{\prime}, V^{\prime}\right)\right|_{\tau^{\prime}=0}=(1.7,0)$

Fig.5 Transient deformation for case (iv) $\quad\left(\lambda>0, \alpha^{\prime}=0.1, \delta^{\prime}=0.2\right)$

Figure 5 shows the transient vibration of the beam for the case (iv), where the temperature is higher than the buckling temperature and the electric voltage is smaller than a critical value. From Fig.5, it is found that the beam vibrates, decays and tends to the final deflection $U_{5}^{\prime}$ (corresponding to $U_{5}$ ) or $U_{6}^{\prime}$ (corresponding to $U_{6}$ ) depending on the initial condition.

In order to examine the effect of the initial condition on the transient vibration for case (iv), we show the transient vibration for various initial conditions in Fig.6 in conjunction with the separatrix, that is, the orbit that passes through the unstable equilibrium point $U_{7}^{\prime}$ (corresponding to $\left.U_{7}\right)$ and disregards the effect of damping $\left(\delta^{\prime}=0\right)$, shown by dotted line.

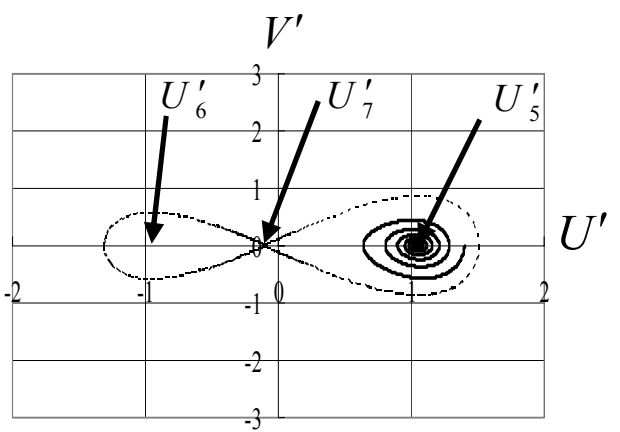

(a) $\left.\left(U^{\prime}, V^{\prime}\right)\right|_{\tau^{\prime}=0}=(1.4,0)$

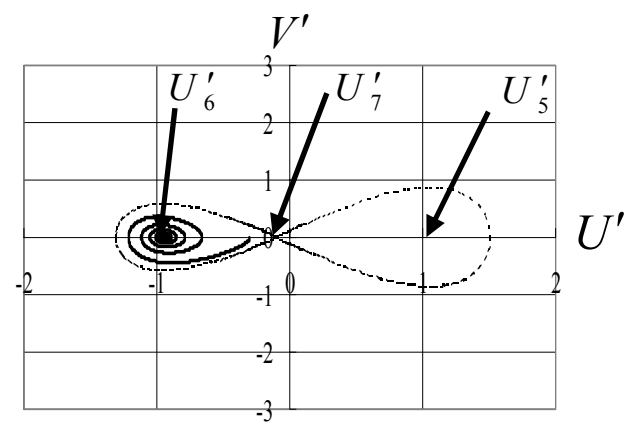

(b) $\left.\left(U^{\prime}, V^{\prime}\right)\right|_{\tau^{\prime}=0}=(-0.3,0)$ 


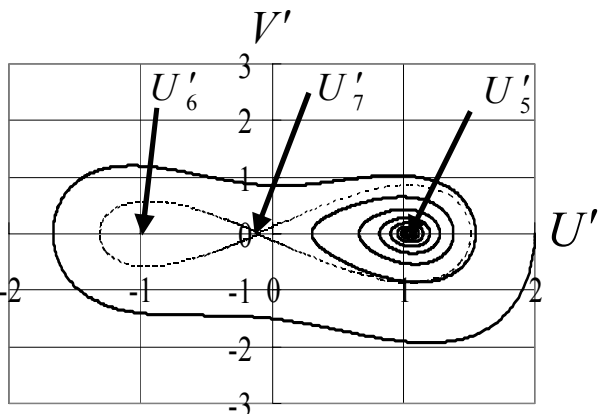

(c) $\left.\left(U^{\prime}, V^{\prime}\right)\right|_{\tau^{\prime}=0}=(2,0)$

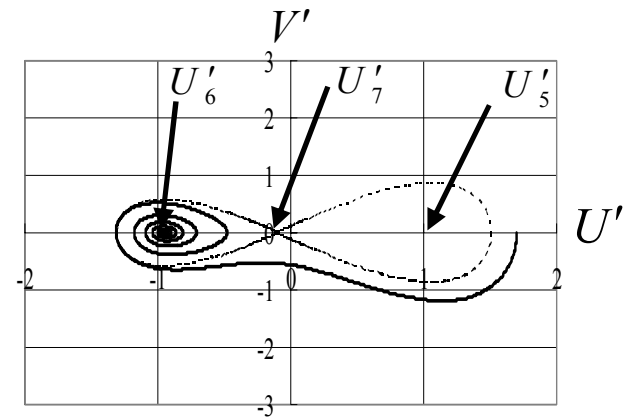

(d) $\left.\left(U^{\prime}, V^{\prime}\right)\right|_{\tau^{\prime}=0}=(1.7,0)$

Fig.6 Transient deformation for case (iv) $\quad\left(\lambda>0, \alpha^{\prime}=0.1, \delta^{\prime}=0.2\right)$

From Figs.6(a)and(b), it is found that, when the initial deformation is inside the branch of the separatrix that encircles a stable equilibrium point, the beam tends to the point. From Figs.6(c)and(d), it is found that, when the initial deformation is outside the separatrix, deformation reaches to a branch and tends to the equilibrium point enclosed by the branch.

Then, the effect of damping on the transient deformation for case (iv) is shown in Fig.7, where solid and dotted lines denote the orbit and separatrix, respectively.

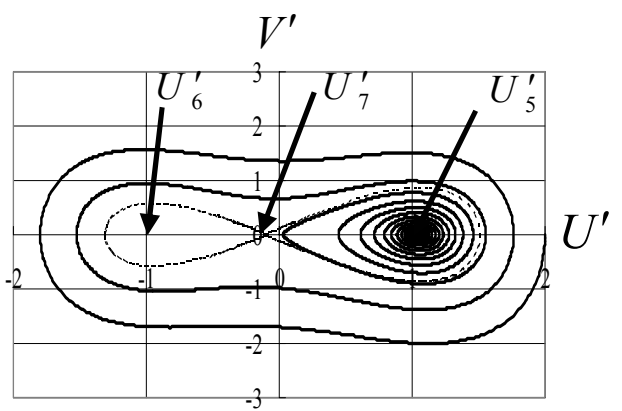

(a) $\delta^{\prime}=0.1$

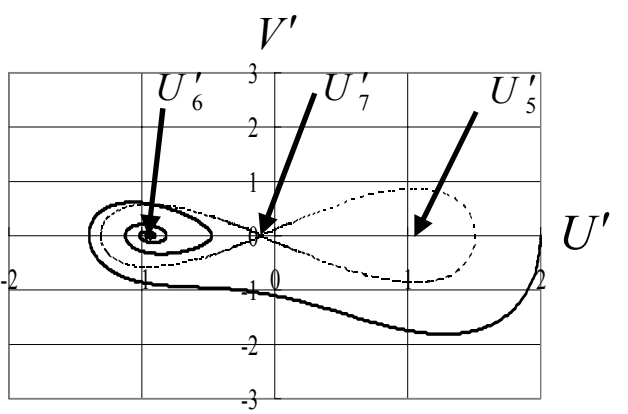

(b) $\delta^{\prime}=0.4$

Fig.7 Transient deformation for case (iv) $\quad\left(\lambda>0, \alpha^{\prime}=0.1,\left.\left(U^{\prime}, V^{\prime}\right)\right|_{\tau^{\prime}=0}=(2,0)\right)$

From Fig.7, it is found that the damping factor $\delta^{\prime}$ affects on the final deflection and that, depending on the factor, the deformation reaches to a branch and tends to the equilibrium point enclosed by the branch.

Finally, the effect of the electric voltage on the transient deformation is shown in Fig.8.

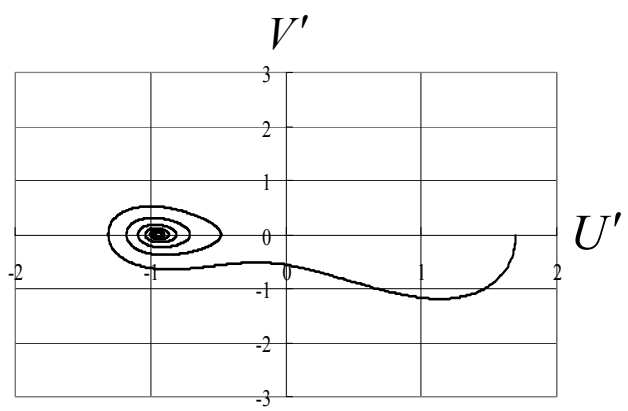

(a) $\quad \alpha^{\prime}=0.1$

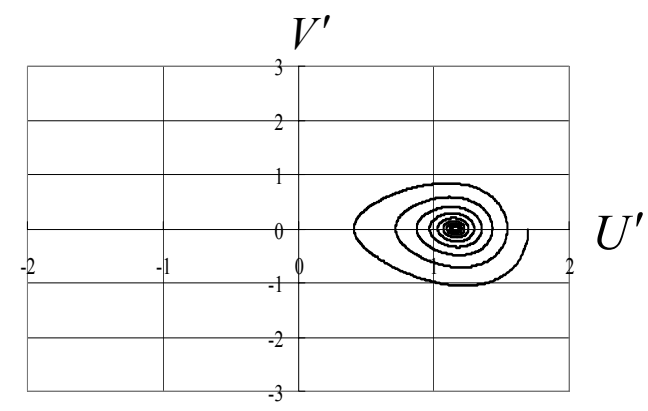

(b) $\quad \alpha^{\prime}=0.4$

Fig.8 Transient deformation $\quad\left(\lambda>0, \delta^{\prime}=0.2,\left.\left(U^{\prime}, V^{\prime}\right)\right|_{\tau^{\prime}=0}=(1.7,0)\right)$

From Fig.8, it is found that desired final deformation can be achieved by applying electric voltage appropriately, which is of great importance in practical viewpoint. 


\section{Conclusion}

We analyze the nonlinear transient behavior of a piezothermoelastic laminate with damping effect considered, including the dynamic deflection deviated arbitrarily from the equilibrium state. As an analytical model, we consider a rectangular laminated beam composed of fiber-reinforced laminate and piezoelectric layers subjected to mechanical, thermal and electrical loads as disturbances or as intended control procedures with both ends simply-supported. Nonlinear large deformation of the laminate is analyzed based on the von Kármán strains and the classical laminate theory. As a result, it is found that the dynamic deflection of the laminate is governed by the equation for a polynomial oscillator. According to the equation, the following quantities are obtained: (1) the buckling temperature due to in-plane thermal and electrical loads; (2) the static large deflection due to combined in-plane and anti-plane loads; (3) the transient large deflection deviated arbitrarily from the static equilibrium; (4) transient large deformation with damping effect considered due to mechanical, thermal and electrical loads. From the results for in-plane thermal and electrical loads, it is found that the buckling temperature can be controlled by appropriate application of the electric voltage to the piezoelectric layers. From the results for the transient large deformation, it is found that the final deformation of the beam is governed by the separatrix, that is, the orbit that passes through the saddle point and disregards the effect of damping and that appropriate application of the electric voltage to the piezoelectric layers can govern the final deformation of the beam.

\section{References}

(1) Mukherjee, A. and Chaudhuri, A.S., Piezolaminated beams with large deformations, International Journal of Solids and Structures, Vol. 39 (2002), pp. 4567-4582.

(2) Shen, H.S., Postbuckling of Shear Deformable Laminated Plates with Piezoelectric Actuators under Complex Loading Conditions, International Journal of Solids and Structures, Vol. 38 (2001), pp. 7703-7721.

(3) Shen, H.S., Postbuckling of Laminated Cylindrical Shells with Piezoelectric Actuators under Combined External Pressure and Heating, International Journal of Solids and Structures, Vol. 39 (2002), pp. 4271-4289.

(4) Tzou, H.S. and Zhou, Y., Dynamics and Control of Nonlinear Circular Plates with Piezoelectric Actuators, Journal of Sound and Vibration, Vol. 188 (1995), pp. 189-207.

(5) Tzou, H.S. and Zhou, Y.H., Nonlinear Piezothermoelasticity and Multi-Field Actuations, Part 2, Control of Nonlinear Deflection, Buckling and Dynamics, Journal of Vibration and Acoustics, Transactions of the ASME, Vol. 119 (1997), pp. 382-389.

(6) Ishihara, M. and Noda, N., Non-Linear Dynamic Behavior of a Piezothermoelastic Laminated Plate with Anisotropic Material Properties, Acta Mechanica, Vol. 166 (2003), pp. 103-118.

(7) Ishihara, M. and Noda, N., Non-Linear Dynamic Behavior of a Piezothermoelastic Laminate Considering the Effect of Transverse Shear, Journal of Thermal Stresses, Vol. 26 (2003), pp. 1093-1112.

(8) Ishihara, M. and Noda, N., Non-Linear Dynamic Behaviour of a Piezothermoelastic Laminate, Philosophical Magazine, Vol. 85 (2005), pp. 4159-4179.

(9) Chia, C.Y., Nonlinear Analysis of Plates (McGraw-Hill, New York, 1980), p. 14.

(10) C.A.J. Fletcher, Computational Galerkin Method, Chapter 1 (Springer, New York, 1984), pp. 1-71.

(11) S. Wiggins, Introduction to Applied Nonlinear Dynamical Systems and Chaos, Chapter 1 (Springer, New York, 1990), pp. 1-192. 